\title{
FUNCTIONAL EXERCISE FOR ADULTS WITH CHRONIC NONSPECIFIC LOW BACK PAIN: RANDOMIZED CONTROLLED CLINICAL TRIAL
}

Emilia Moreira (Universidade Federal de São Paulo, São Paulo, SP, Brasil), Anamaria Jones (Universidade Federal de São Paulo, São Paulo, SP, Brasil), Eider Silva Lima (Universidade Federal de São Paulo, São Paulo, SP, Brasil), Fabio Jennings (Universidade Federal de São Paulo, São Paulo, SP, Brasil), Jamil Natour (Universidade Federal de São Paulo, São Paulo, SP, Brasil)

\section{BACKGROUND}

Low back pain is the most prevalent musculoskeletal condition and one of the most common causes of disability. Exercise therapy is part of the recommendation for the treatment of chronic non-specific disease. Functional exercise may be an alternative for the treatment of patients with low back pain, no studies assessing its effectiveness were found.

Thefore, the purpose of this study as to assess the effectiveness of the functional exercise program for pain, functional capacity, quality of life, kinesiophobia, medication consumption and patient satisfaction in adults with chronic nonspecific low back pain.

\section{MATERIALS AND METHODS}

A randomized, single-blind, controlled clinical trial with intention-to-treat analysis and 24-week follow-up was performed. Eighty-four patients were randomly assigned to an experimental group (EG) or control group (CG). The EG patients participated in the functional exercises program performed twice a week for twelve weeks. The functional exercise program was composed by global exercise that worked the group of muscles of the trunk and lower and upper limbs with progression every 4 weeks. The two groups received an informative class on the disease and were advised to use analgesic if necessary. Primary outcome was low back pain measured by numeric rating scale (NRS). Secondary outcomes included was functional capacity by Oswestry and Roland Morris questionnaires, 6-minute walk and TUG tests, fearavoidance (FABQ), general health (SF-36), medications consumption and satisfaction with treatment (Likert scale). Evaluations were performed at baseline, after 6, 12 (end of intervention) and 24 weeks by a blind evaluator.

\section{RESULTS}

The groups were homogeneous for all parameters at baseline. Compared with the CG, the EG significantly improved pain $(p<0.001)$, functional capacity (Oswestry, Roland Morris $(p<0.001)$ and TUG $(p=0.005)$ ), FABQ $(p<0.001)$ and most of the SF-36 parameters.

\section{CONCLUSION}

The functional exercise program was effective in improving pain, functional capacity, kinesiophobia, general health and perception of effort after walking test in adults with chronic nonspecific low back pain. 